\title{
The Evaluation of Determinants by Expansion by Minors and the General Problem of Substitution*
}

\author{
By W. M. Gentleman and S. C. Johnson**
}

\begin{abstract}
In this paper, we consider a class of substitution strategies which correspond to various minor expansion algorithms, and show that the usual expansion by minors, a row at a time, is optimal in this class.
\end{abstract}

Motivation. One of the least understood aspects of symbolic formula manipulation is the problem of substitution, particularly the merits of various strategies. A specific example, where substitution is complicated enough to be interesting and where theoretical results can be obtained, is the problem of evaluating a determinant by substitution into the Laplace expansion.

Introduction. One obvious possibility for evaluating determinants is substitution into the Laplace expansion, regarded as a polynomial form of $n$ ! terms in $n^{2}$ indeterminates. The obvious way to substitute into this form requires $(n-1)$ multiplications per term, a total of $(n-1) n$ ! multiplications. Many of these multiplications are redundant, and no advantage is taken of any collection of like terms. Some sort of parenthesizing and grouping of terms, as in Horner's rule, is clearly indicated.

Many such groupings are possible. For example, when $n=4$, the 4 ! terms

$$
\begin{aligned}
& x_{11} x_{22} x_{33} x_{44}-x_{11} x_{22} x_{34} x_{43}-x_{11} x_{23} x_{32} x_{44}+x_{11} x_{23} x_{34} x_{42} \\
& -x_{11} x_{24} x_{33} x_{42}+x_{11} x_{24} x_{32} x_{43}-x_{12} x_{21} x_{33} x_{44}+x_{12} x_{21} x_{34} x_{43} \\
& +x_{12} x_{23} x_{31} x_{44}-x_{12} x_{23} x_{34} x_{41}+x_{12} x_{24} x_{33} x_{41}-x_{12} x_{24} x_{31} x_{43} \\
& +x_{13} x_{21} x_{32} x_{44}-x_{13} x_{21} x_{34} x_{42}-x_{13} x_{22} x_{31} x_{44}+x_{13} x_{22} x_{34} x_{41} \\
& +x_{13} x_{24} x_{31} x_{42}-x_{13} x_{24} x_{32} x_{41}+x_{14} x_{21} x_{33} x_{42}-x_{14} x_{21} x_{32} x_{43} \\
& +x_{14} x_{22} x_{31} x_{43}-x_{14} x_{22} x_{33} x_{41}-x_{14} x_{23} x_{31} x_{42}+x_{14} x_{23} x_{32} x_{41}
\end{aligned}
$$

can be written, among other ways, as

$$
\begin{aligned}
& \left(x_{11} x_{22}-x_{12} x_{21}\right)\left(x_{33} x_{44}-x_{34} x_{43}\right)-\left(x_{11} x_{23}-x_{13} x_{21}\right)\left(x_{32} x_{44}-x_{34} x_{42}\right) \\
& +\left(x_{11} x_{24}-x_{14} x_{21}\right)\left(x_{32} x_{43}-x_{33} x_{42}\right)+\left(x_{12} x_{23}-x_{13} x_{22}\right)\left(x_{31} x_{44}-x_{34} x_{41}\right) \\
& -\left(x_{12} x_{24}-x_{14} x_{22}\right)\left(x_{31} x_{43}-x_{33} x_{41}\right)+\left(x_{13} x_{24}-x_{14} x_{23}\right)\left(x_{31} x_{42}-x_{32} x_{41}\right)
\end{aligned}
$$

Received June 8, 1973.

AMS (MOS) subject classifications (1970). Primary 15A15, 68A15, 68A20.

Key words and phrases. Substitution, expansion by minors, determinants.

* This work was supported in part by the National Research Council of Canada Grant A7407.

** This author was at the University of Waterloo on leave from Bell Telephone Laboratories, Murray Hill, New Jersey while working on this paper.

Copyright @ 1974, American Mathematical Society 
or again as

$$
\begin{aligned}
& \left(a_{1} x_{33}-a_{2} x_{32}+a_{4} x_{31}\right) x_{44}-\left(a_{1} x_{34}-a_{3} x_{32}+a_{5} x_{31}\right) x_{43} \\
& +\left(a_{2} x_{34}-a_{3} x_{33}+a_{6} x_{31}\right) x_{42}-\left(a_{4} x_{34}-a_{5} x_{33}+a_{6} x_{32}\right) x_{41}
\end{aligned}
$$

where

$$
\begin{array}{ll}
a_{1}=\left(x_{11} x_{22}-x_{12} x_{21}\right), & a_{2}=\left(x_{11} x_{23}-x_{13} x_{21}\right), \\
a_{3}=\left(x_{11} x_{24}-x_{14} x_{21}\right), & a_{4}=\left(x_{12} x_{23}-x_{13} x_{22}\right), \\
a_{5}=\left(x_{12} x_{24}-x_{14} x_{22}\right), & a_{6}=\left(x_{13} x_{24}-x_{14} x_{23}\right) .
\end{array}
$$

When such groupings are tried, the productive ones simply turn out to be statements of the well-known general combinatorial definition of determinants, expanding by minors.

Definition. The determinaht of a square matrix of order 1 is the entry in that matrix. The determinant of a matrix of order $n$ can be found by choosing $m$ less than $n$, and selecting $m$ columns from the original determinant. Consider then each of the $\left(\begin{array}{l}n \\ m\end{array}\right)$ ways that $m$ rows can be chosen from the original determinant. Each row choice defines two submatrices: one of order $m$ corresponding to the chosen rows and columns, and one of order $n-m$ corresponding to the complementary sets. The original determinant is the sum (with appropriate signs), over all row choices, of the product of these two smaller determinants (minors).

Any direct implementation of this recursive definition is very inefficient, as it leads to the repeated recalculation of small minors. Nonrecursive implementations, however, can be obtained in the following manner: Consider any rooted binary (bifurcating) tree with $n$ leaves labelled 1 through $n$ (for example, Figures 1 and 2 below). With each node in the tree (including the leaves), we associate a set of minors, as follows: If the subtree rooted at the given node has $m$ leaves, we associate with this node the set of $\left(\begin{array}{l}n \\ m\end{array}\right)$ minors obtained by all possible choices of $m$ rows, with the column indices given by the $m$ leaf names. We note the following simple properties of these minor sets:

(1) The minor set for the root of the tree has a single element, the determinant.

(2) The minor set of leaf $k$ has $m$ elements, which are simply the elements of column $k$ of the matrix.

(3) Using the above combinatorial definition of determinants, we can compute the minor set of a given node from the minor sets of its two immediate descendents. In this way, every tree corresponds to a (nonrecursive) algorithm for computing the determinant by minor expansion.

As a specific example, consider the classical method of expansion by column minors. In this method, one computes all the minors determined by two particular columns, then uses these to obtain all the minors determined by these columns plus one additional column, then uses these to obtain all the minors determined by four columns, etc. This corresponds to a tree, such as in Figure 2, where each node except leaves has one descendent which is a leaf. In fact, the following theorem shows that expansion by column minors is one of the best minor expansions.

THEOREM. The minimum cost (in multiplications) of expanding an $n \times n$ determinant by minors (using any of the above trees) is $n\left(2^{n-1}-1\right)$, which is the cost of expanding by column minors. 


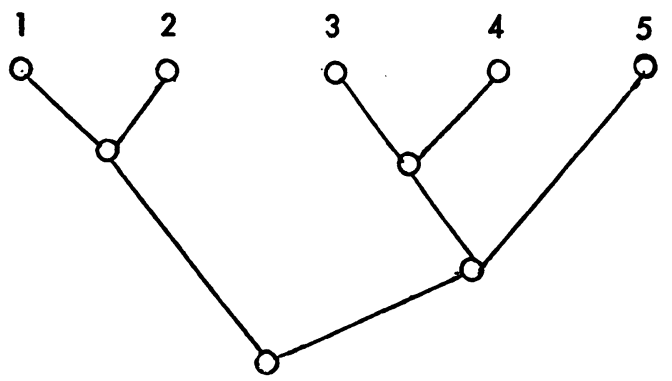

FIGURE 1

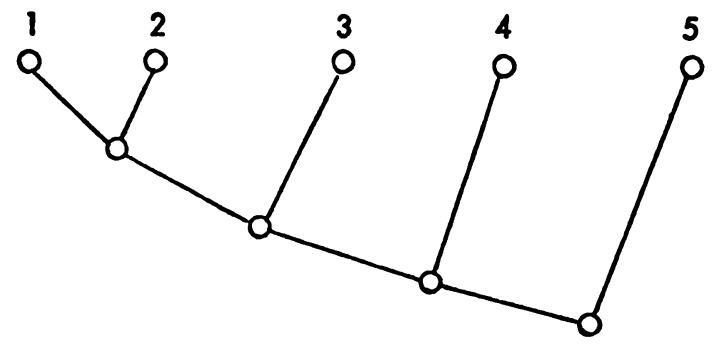

FigURE 2

Proof. Suppose that the subtree rooted at a specific node has $m$ leaves, and its two descendant subtrees have $k$ and $m-k$ leaves, respectively, where $k \leqq m-k$. There are $\left(\begin{array}{l}n \\ m\end{array}\right)$ minors of order $m$ in the minor set of this node; each is obtained by forming and summing $\left(\begin{array}{c}m \\ k\end{array}\right)$ products of minors from the minor sets of the two descendent nodes. The cost (in multiplications) of producing all $\left(\begin{array}{l}n \\ m\end{array}\right)$ minors is thus $\left(\begin{array}{l}n \\ m\end{array}\right)\left(\begin{array}{l}m \\ k\end{array}\right)$, plus the cost of obtaining the minors defined by each subtree.

The minor set of this node could obviously be computed by expansion by column minors. From the preceding remarks, this would cost $\sum_{i=2}^{m}\left(\begin{array}{l}n \\ i\end{array}\right)\left(\begin{array}{l}i \\ 1\end{array}\right)$ (since $k=1$ at each of the $m-1$ stages). For $m=n$, i.e., for computing the original determinant by expansion by column minors, this can be summed in closed form:

Cost (column minors) $=\sum_{i=2}^{n}\left(\begin{array}{l}n \\ j\end{array}\right)\left(\begin{array}{l}j \\ 1\end{array}\right)=\sum_{i=2}^{n} \frac{n ! j}{f !(n-j) !}$

$$
=n \sum_{i=2}^{n} \frac{(n-1) !}{(j-1) !(n-j) !}=n \sum_{j=1}^{n-1}\left(\begin{array}{c}
n-1 \\
j
\end{array}\right)=n\left(2^{n-1}-1\right) .
$$

We will prove this is minimal over all binary trees by inductively showing that to compute the minor set of a node covering $m$ leaves, the minimal cost is $\sum_{i=2}^{m}\left(\begin{array}{c}n \\ i\end{array}\right) j$. This is clearly true if $m=2$ or even $m=3$, since there only is one possible tree. Assume it is true for $2,3, \cdots, m-1$. The minimum cost to cover $m$ columns is thus, for some $k$,

$$
\left(\begin{array}{l}
n \\
m
\end{array}\right)\left(\begin{array}{l}
m \\
k
\end{array}\right)+\sum_{j=2}^{k}\left(\begin{array}{l}
n \\
j
\end{array}\right) j+\sum_{j=2}^{m-k}\left(\begin{array}{l}
n \\
j
\end{array}\right) j
$$

Our theorem is proved if we show that this cost is minimized at $k=1$. The difference between the cost for $k$ and the cost for $k-1$ is 


$$
\left(\begin{array}{l}
n \\
m
\end{array}\right)\left(\begin{array}{l}
m \\
k
\end{array}\right)-\left(\begin{array}{l}
n \\
m
\end{array}\right)\left(\begin{array}{c}
m \\
k-1
\end{array}\right)+\left(\begin{array}{l}
n \\
k
\end{array}\right) k-\left(\begin{array}{c}
n \\
m-k+1
\end{array}\right)(m-k+1) .
$$

We will show that this is nonnegative for $k \geqq 2$; thus $k=1$ is minimal. Rewriting the above expression, what we want to prove is

$$
\left(\begin{array}{l}
n \\
m
\end{array}\right)\left[\left(\begin{array}{l}
m \\
k
\end{array}\right)-\left(\begin{array}{c}
m \\
k-1
\end{array}\right)\right]+\left(\begin{array}{l}
n \\
k
\end{array}\right) k-\left(\begin{array}{c}
n \\
m-k+1
\end{array}\right)(m-k+1) \geqq 0
$$

i.e.,

$$
\begin{aligned}
& \frac{n !}{m !(n-m) !} {\left[\frac{m !}{k !(m-k) !}-\frac{m !}{(k-1) !(m-k+1) !}\right] } \\
&+\frac{n ! k}{k !(n-k) !}-\frac{n !(m-k+1)}{(m-k+1) !(n-m+k-1) !} \\
&= \frac{n !}{(n-m) !(k-1) !(m-k) !}\left[\frac{1}{k}-\frac{1}{m-k+1}+\frac{(n-m) !(m-k) !}{(n-k) !}\right. \\
&\left.\quad-\frac{(n-m) !(k-1) !}{(n-m+k-1) !}\right] \geqq 0
\end{aligned}
$$

and, since the factor outside the square brackets is clearly positive, we need only consider the quantity within, which can be written as

$$
\frac{n-2 k+1}{k(m-k+1)}+\left(\begin{array}{c}
n-k \\
n-m
\end{array}\right)^{-1}-\left(\begin{array}{c}
n-m+k-1 \\
n-m
\end{array}\right)^{-1}
$$

It is convenient to rewrite this in terms of $s=n-m$ and $t=m-2 k$ as

$$
D(k, s, t)=\frac{t+1}{k(k+t+1)}+\left(\begin{array}{c}
k+t+s \\
s
\end{array}\right)^{-1}-\left(\begin{array}{c}
k+s-1 \\
s
\end{array}\right)^{-1}
$$

which we must prove nonnegative for $k \geqq 2, s \geqq 0, t \geqq 0$. We will do this by considering five cases.

Case I. $s=0, t \geqq 0, k \geqq 2$.

$$
D(k, s, t)=\frac{t+1}{k(k+t+1)}+1-1=\frac{t+1}{k(k+t+1)}
$$

which is clearly positive.

Case II. $s=1, t \geqq 0, k \geqq 2$.

$$
D(k, s, t)=\frac{t+1}{k(k+t+1)}+\frac{1}{k+t+1}-\frac{1}{k}=0 .
$$

Case III. $s=2, t \geqq 0, k \geqq 2$.

$$
\begin{aligned}
D(k, s, t) & =\frac{t+1}{k(k+t+1)}+\left(\begin{array}{c}
k+t+2 \\
2
\end{array}\right)^{-1}-\left(\begin{array}{c}
k+1 \\
2
\end{array}\right)^{-1} \\
& =\frac{t+1}{k(k+t+1)}+\frac{2}{(k+t+1)(k+t+2)}-\frac{2}{k(k+1)} \\
& =\frac{(k-1) t^{2}+\left(k^{2}-3\right) t+(k-2)(k+1)}{k(k+1)(k+t+1)(k+t+2)}
\end{aligned}
$$


and, since the numerator is a polynomial in $t$ which, for any $k \geqq 2$, has nonnegative coefficients, it is nonnegative for $t \geqq 0$.

Case IV. $s \geqq 3, t=0, k \geqq 2$.

$$
\begin{aligned}
D(k, s, t) & =\frac{1}{k(k+1)}+\left(\begin{array}{c}
k+s \\
s
\end{array}\right)^{-1}-\left(\begin{array}{c}
k+s-1 \\
s
\end{array}\right)^{-1} \\
& =\frac{1}{k(k+1)}+\frac{k ! s !}{(k+s) !}-\frac{(k-1) ! s !}{(k+s-1) !} \\
& =\frac{1}{k(k+1)}+\frac{k ! s !}{(k+s) !}\left[1-\frac{k+s}{k}\right]=\frac{1}{k(k+1)}-\frac{s k ! s !}{k(k+s) !} \\
& =\frac{s^{2}}{k(k+1)}\left[\frac{1}{s^{2}}-\frac{(k+1) !(s-1) !}{(k+s) !}\right] .
\end{aligned}
$$

Thus, it suffices to show $\left(\begin{array}{c}k++ \\ k+1\end{array}\right) \geqq s^{2}$. But this follows since

$$
\left(\begin{array}{l}
k+s \\
k+1
\end{array}\right)-s^{2} \geqq\left(\begin{array}{c}
s+2 \\
3
\end{array}\right)-s^{2}=\frac{(s+2)(s+1) s-6 s^{2}}{6}=\frac{s(s-1)(s-2)}{6}
$$

which is nonnegative for $s \geqq 2$.

Case V. $s \geqq 3, t \geqq 1, k \geqq 2$.

$$
D(k, s, t) \geqq \frac{2}{k(k+2)}-\left(\begin{array}{c}
k+s-1 \\
s
\end{array}\right)^{-1} \geqq \frac{2}{k(k+2)}-\left(\begin{array}{c}
k+2 \\
3
\end{array}\right)^{-1} .
$$

But

$$
\begin{aligned}
& \frac{2}{k(k+2)}-\left(\begin{array}{c}
k+2 \\
3
\end{array}\right)^{-1} \\
& \quad=\frac{2}{k(k+2)}-\frac{6}{k(k+1)(k+2)}=\frac{2}{k(k+2)}\left[1-\frac{3}{k+1}\right]
\end{aligned}
$$

which for $k \geqq 2$ is nonnegative. Q.E.D.

Note. The above proof shows expanding by column minors to be optimal, but it is not the only optimal scheme. The order in which the columns are introduced is arbitrary, and, moreover, the role of columns and rows can obviously be interchanged.

Conclusions. At first glance, the theorem above seems rather negative; everyone knows that expansion by column minors is too expensive to be a practical algorithm, and we have shown no other form of minor expansion is better. However, the wellknown expense of minor expansion is an asymptotic statement, and for small $n$ (less than 6) minor expansion is actually cheaper than Gaussian elimination. Moreover, the assertion about expense is based on an operation count that assumes all multiplications are equally expensive; in computations of symbolic algebra this is far from true, and determinant evaluation by minors is often the best way. Thus, the study of minor expansion as a practical algorithm is relevant, and, in this context, the result is pleasantly surprising - surprising, because we usually expect algorithms associated with balanced trees to be preferable to those associated with unbalanced 
ones, whereas here the most unbalanced tree is best, and pleasant because expansion by column minors is certainly the simplest minor expansion to implement. Moreover, we get an extra benefit: the implication of the results on polynomial powering [1] is that it is generally better, given the choice, to perform operations between one derived quantity and one quantity from the original problem rather than to perform operations between two derived quantities, and of course that is what distinguishes expansion by column minors. (The advantages can be quantified here too, by only slight changes in the proof above.)

What does all this have to do with the general problem of substitution into an arbitrary form? In determinant evaluation, we fortunately had a set of groupings that we could study theoretically. In the case of an arbitrary form, it is not clear what strategy to use in grouping terms. Horner's rule is satisfactory if the form is a univariate polynomial, but if the form is multivariate, the obvious recursive generalization of Horner's rule (regarding polynomials in $n-j$ variables as polynomials in one variable with coefficients that are polynomials in $n-j-1$ variables) is unsatisfactory since it can involve recomputing common subexpressions. On the other hand, it is most unlikely that any practical strategy can be more complicated then augmenting the obvious recursive scheme with a pattern match to recognize common subexpressions. Such strategy has the advantage for symbolic computation referred to above, i.e., that all operations are performed between a derived quantity and one from the original problem; but what other assurance have we that it is reasonable? One such is that this strategy, applied to the Laplace expansion, leads to what is effectively expansion by column minors.

Department of Applied Analysis and Computer Science

University of Waterloo

Waterloo, Ontario, Canada

Bell Telephone Laboratories

Murray Hill, New Jersey 07974

1. W. M. Gentleman, "Optimal multiplication chains for computing a power of a symbolic polynomial," Math Comp., v. 26, 1972, pp. 935-939.

2. SIR THOMAs MUIR, The Theory of Determinants in the Historical Order of Development, Macmillan, London, 1906-1920. 